\title{
Monte Carlo Calibrated Drift-Diffusion Simulation of Short Channel HFETs
}

\author{
A. ASENOV*, S. BABIKER, S. P. BEAUMONT and J. R. BARKER \\ Nanoelectronics Research Centre, Department of Electronics and Electrical Engineering, \\ Glasgow University, Glasgow G12 8LT, Scotland, UK
}

\begin{abstract}
In this paper we present a methodology to use drift diffusion (DD) simulations in the design of short channel heterojunction FETs (HFETs) with well pronounced velocity overshoot. In the DD simulations the velocity overshoot in the channel is emulated by forcing the saturation velocity in the field dependent mobility model to values corresponding to the average velocity in the channel obtained from Monte Carlo (MC) simulation. To illustrate our approach we compare enhanced DD and MC simulation results for a pseudomorphic HEMTs with $0.12 \mu \mathrm{m}$ channel length, which are in good agreement. The usefulness of the described methodology is illustrated in a simulation example of self aligned gamma gate pseudomorphic HEMTs. The effect of the gamma gate shape and the self aligned contacts on the overall device performance has been investigated.
\end{abstract}

Keywords: Numerical simulation, overshot, heterojunction FETs, drift-diffusion approach

\section{INTRODUCTION}

Commercial device simulators like MEDICI [1] and BLAZE [2] are flexible, fast and work in a user friendly environment. Employing finite element approach they can describe accurately the complex geometry of modern short recess gate heterojunction FETs (HFETs). Interface charge, surface states, and deep levels can be eventually included in the simulations and the self heating can be treated through coupling to the heat flow equation. The transient algorithms are stable and allow for large time steps. Frequency domain analysis is readily available. External circuit elements like contact and gate resistances, pad inductances and capacitances can be included in the simulations and in the $r f$ analyses. Unfortunately the drift-diffusion (DD) approach, which is at the heart of such simulators, cannot predict the velocity overshoot responsible for the high performance of many short channel HEFTs [3]. The hydrodynamic (HD) options offered as extensions to the DD engines of the above simulators deal with the overshoot but slow down the simulations

*Corresponding author. Tel: +44 141330 5233, Fax: + 44141330 4907, E-mail: A. Asenov@elec.gla.ac.uk. 
and often have convergence and parameter indentification problems. The ensemble Monte Carlo (MC) method, usually implemented in inhouse software [4], is still computationally expensive and cannot match all the features of the DD commercial simulators. particularly their speed.

In this paper we describe a methodology for using MC calibrated DD simulation with enhanced channel velocity in the design and optimisation of short channel HFETs including HEMTs and strained Si channel SiGe MODFETs. The DD simulations are calibrated with respect to our finite element Monte Carlo simulator $\mathrm{H} 2 \mathrm{~F}$ [5]. The usefulness of this approach is illustrated in simulation exampies of self aligned gamma gate pseudomorphic HEMTs.

\section{EMULATION OF THE OVERSHOOT}

The enhanced DD approach is based on the observation from MC simulations that in many short channel $(0.1-0.2 \mu \mathrm{m}$ gate) HFETs the velocity overshoot extends along the whole high field channel region. The average velocity profile in the channel obtained from MC simulation can be emulated by increasing the saturation velocity $v_{s}$ in a simple, three parameter, silicon-type field dependent mobility model [6] with velocity $v$ is given by

$$
v=\frac{\mu_{0} E}{\left[1+\left(\frac{\mu_{0} E}{v_{s}}\right)^{b}\right]^{\frac{1}{b}}}
$$

where $E$ is the electric field, $\mu_{0}$ is the low field mobility and $b$ is a model parameter. The calibration is done by adjusting the three parametes $\mu_{0}, v_{s}$ and $b$ in order to match the MC velocity profile in the source and in the channel region for a bias point in the middle of the uselful part of the device characteristics. In most of the cases this is enough to achieve satisfactory agreement between the MC and the DD simulation results over the whole range of applied voltages of interest.
Figure 1 illustrates the velocity along the channel of a $0.12 \mu$ gate length pseudomorphic HEMT with $22 \mathrm{~nm}$ gate-to-channel separation, 50 $\mathrm{nm}$ recess offset and delta doping in the supply layer. The device is described in more details elsewhere [7]. The velocity profile obtained from DD MEDICI simulation with $v_{s}$ forced to $2.8 \times 10^{7} \mathrm{~cm} / \mathrm{s}, \quad \mu_{0}=5000 \mathrm{~cm}^{2} \mathrm{v}^{-1} \mathrm{~s}^{-1}$ and $b=2$ matches well with the MC velocity profile in the source region and in the channel. In the drain region, where most of the particle in the MC simulation are in the L-valley, the DD simulation overestimates the velocity and hence underestimates the drain resistance. This however does not affect seriously the simulated $r f$ performance since the drain resistance has usually a weak influence on the measured and extracted $s$-parameters.

\section{COMPARISON OF DD AND MC DC AND RF RESULTS}

In order to justify the use of the enhanced DD approach in practical short channel HFET device simulation and design we compere the $d c$ and the $r f$ results obtained from both DD and MC simulations. The comparison is based on the same

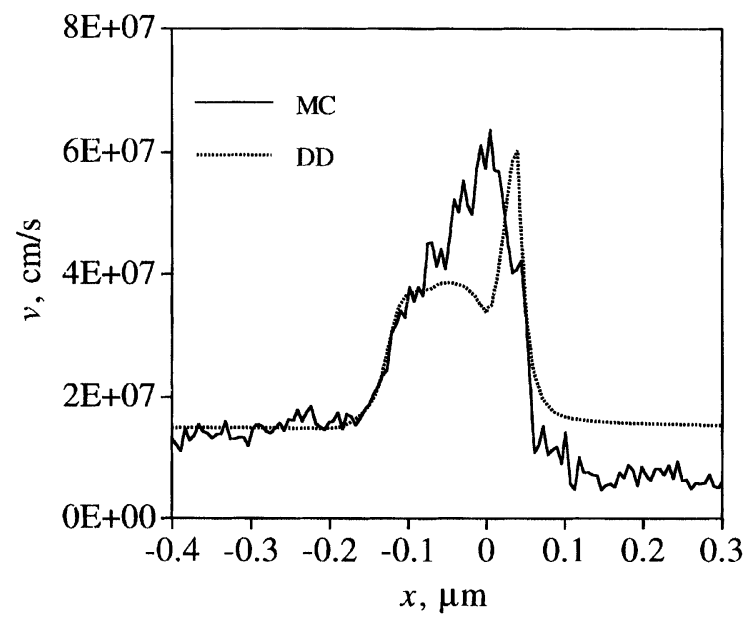

FIGURE 1 Average velocity in the channel of a $0.12 \mu \mathrm{m}$ pHEMT obtained from DD and MC simulation at $V_{G}=0 \mathrm{~V}$ and $V_{D}=1.5 \mathrm{~V}$. 
pseudomorphic HEMTs described in Section 2. Exactly the same simulation geometry is used in both the DD MEDICI's and the MC H2F's simulations. The source and drain contact resistances which cannot be directly included in the MC simulation are also excluded from the DD simulations. The velocity profile is calibrated at one $d c$ bias point corresponding to $V_{G}=0 \mathrm{~V}$ and $V_{D}=1.5 \mathrm{~V}$. The $d c$ ouput characteristics are compared in Figure 2 and are in remarkably good agreement in both the low and in the high drain volatge regions. This is partially due to the fact that the deconfinement which cannot be properly treated in the DD simulation does not affect seriously the operation of the simulated pseudomorphic HEMTs with relatively deep $\left(\operatorname{In}_{0.3-}\right.$ $\mathrm{Ga}_{0.7} \mathrm{As}$ ) channels.

The comparison of the $r f$ results obtained form DD and MC simulation is based on time domain transient technique which is the only available choice in the MC case [8]. Complex two port $y$ parameters are extracted by Fourier transforming the gate and drain current transients in response to small changes in the gate and drain voltages. The frequency dependence of the real and the imaginary parts of $y_{11}$ and $y_{12}$ is plotted in Figure 3. The $y$-parameters extracted from the $\mathrm{DD}$ and $\mathrm{MC}$ transient simulations are in good agreement. This

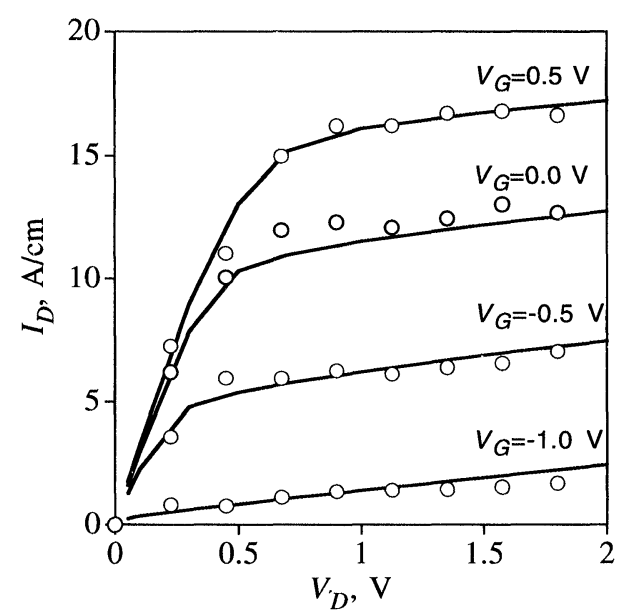

FIGURE 2 Comparison between DD and MC simulated output characteristics of a $0.12 \mu \mathrm{m}$ pHEMT.

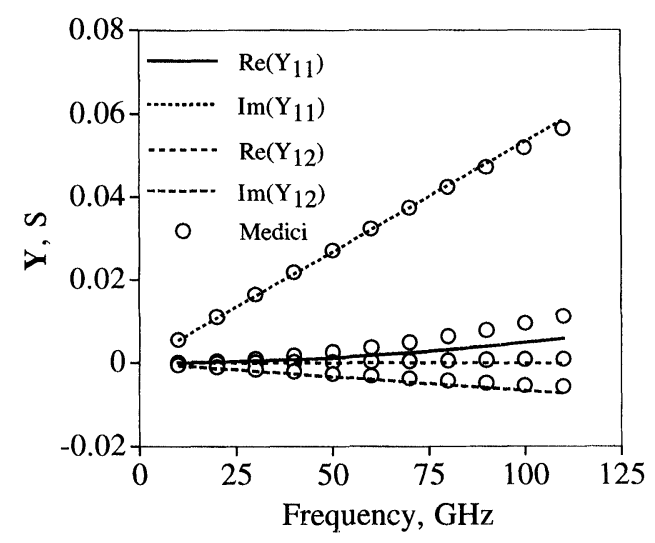

FIGURE 3 Comparison between $Y$ parameters extracted from DD and MC simulation of a $0.12 \mu \mathrm{m}$ pHEMT at $V_{G}=$ $-0.2 \mathrm{~V}$ and $V_{D}=1.5 \mathrm{~V}$. The channel width is $100 \mu \mathrm{m}$.

agreement holds also for the small signal circuit elements and the cut-off frequency $f_{T}$ extracted from the $y$-parameters and for the maximum frequency of oscillations $f_{\max }$ extracted after transforming the $y$-parameters into $s$-parameters.

\section{SELF ALIGNED GAMMA GATE EXAMPLE}

Calibrated DD simulations can be used confidently to investigate device geometry effects which do not affect strongly the lateral field profile in the channel but are important for the $r f$ device performance including the gate and contact shapes, self-alignment, cap layer modification, recess design etc. When the lateral field profile is altered, for example as a result of channel length scaling, the DD simulations have to be recalibrated with respect to a new MC calculation.

We illustrate the described DD approach in the simulation of self aligned gamma gate pHEMTs. The vertical layer structure of these devices is the same as the structure described in Section 2. The profile of the gamma gate and the corresponding solution domain are outlined in Figure 4. In the DD simulation we investigate the effect of the gate overlap $d_{c-r}$ on the drain side of the channel on $f_{T}$ and $f_{\max }$. The results are presented in Figure 5 . 

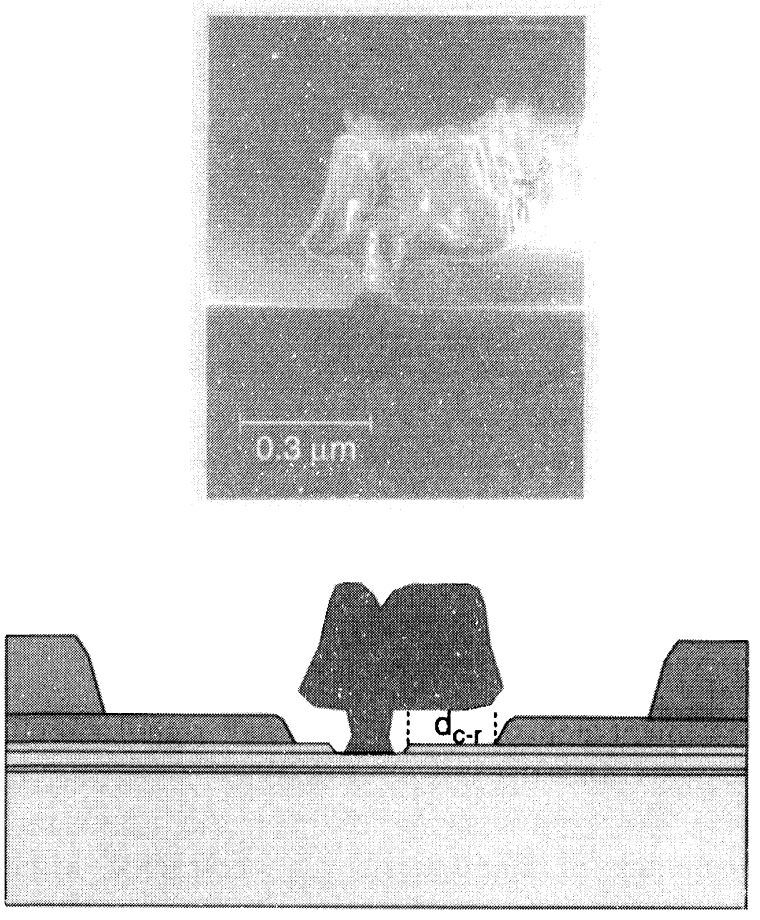

FIGURE 4 Self aligned gamma gate pHEMT.

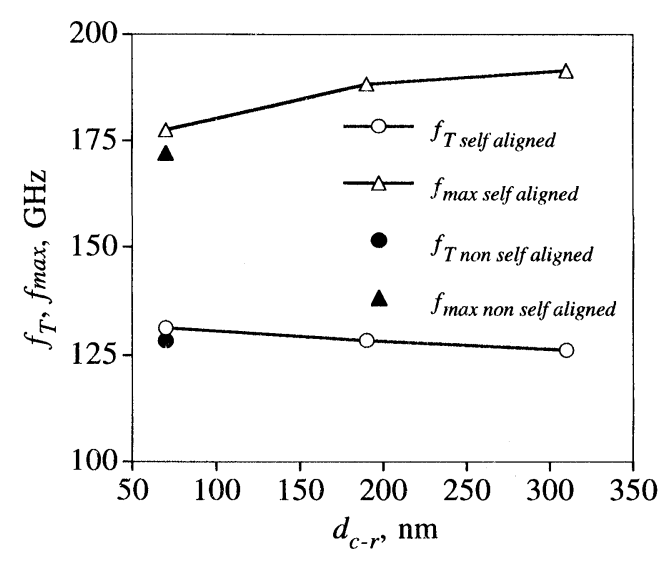

FIGURE 5 Dependence of $f_{T}$ and $f_{\max }$ on the gate overlap $d_{c-r}$ for the self aligned gamma gate pHEMT illustrated in Figure 4 with channel width is $100 \mu \mathrm{m}$, contact resistance $1 \Omega$ and gate resistance $5 \Omega$. DD simulations.

In the self aligned technology a trade-off between the reduced series resistances and the increased gate-to-contacts parasitic capacitances determines the over all device performance. The gamma gate design itself is a trade off between the reduced gate resistance and the increased gate-to-drain parasitic capacitance.

\section{CONCLUSIONS}

The velocity overshoot in the channel of modern short gate length HFETs can be satisfactory emulated in DD simulations by increasing the saturation velocity in a simple silicon type field dependent mobility model. Usually calibration of the DD simulations to one properly selected bias point of the corresponding MC simulations leads to good agreement in the DD and MC $d c$ and $r f$ characteristics over a wide range of applied voltages. Calibrated DD simulations can be used to speed up the design of modern short channel HFETs where geometry related parasitic effects complete with the enhanced channel transport in determining the overall device performance.

\section{Acknowledgements}

The work is supported by EPSRC through the Nanolelectronics Rolling Grant RG/6601BS.

\section{References}

[1] TMA MEDICI,. Version 2.3, Technology Modelling Associates, Inc., 1996.

[2] Silvaco, BL.ASE. Version 4.0.0.R, Silvaco International, 1996.

[3] Babiker, S., Cameron, N., Asenov, A. and Beaumont, S. P. (1996). 'New evidence for velocity overshoot in a $200 \mathrm{~nm}$ Pseudomorphic HEMT', Microelectronics Journal, 27(8), 785-793.

[4] Park, D. H. and Brennan, K. F. (1990). 'Monte Carlo simulation of $0.35 \mu \mathrm{m}$ gate-length $\mathrm{GaAs}$ and InGaAs HEMT', IEEE Trans. Electron Devices, 37(3), 618-628.

[5] Babiker, S., Asenov, A., Barker, J. R. and Beaumont, S. P. (1996). 'Finite element Monte Carlo simulation of recess gate compound FETs', Solid State Electronics, 39(5), $629-635$.

[6] Caughey, D. M. and Thomas, R. E. (1967). 'Carrier mobilities in silicon empirically related to doping and field', IEEE Trans. Electron Devices, 55, 2192-2193.

[7] Cameron, N. I., Murad, S., McLelland, H., Asenov, A., Taylor, M. R. S., Holland, M. C. and Beaumont, S. P. (1996). 'Gate Recess Engineering of Pseudomorphic 
$\mathrm{In}_{0.30} \mathrm{GaAs} / \mathrm{GaAs}$ HEMTs'. Electronics Letters, 32(8), $770-772$.

[8] González, T. and Pardo, D. (1995). 'Monte Carlo determination of the intrinisic small-signal equivalent circuit of MESFET's', IEEE Trans. Electron Devices, 42(4), 605-611.

\section{Authors' Biographies}

Asen Asenov had 10 years industrial experience as a head of the Process and Device Modelling Group in IME-Sofia, developing one of the first integrated process and device CMOS simulators IMPEDANCE. He is currently a Reader in the Department of Electronics and Electrical Engineering, Glasgow University. As a leader of the Device Modelling Group he is involved in the development of 2D and 3D device simulators and their application in the design of compound FETs, SiGe MOSFETs and IGBTs. Other interest include design of parallel algorithms.

Sharief Babiker studied Electrical Engineering in Khartoum University Sudan (1979-1984). During the period 1985-90 he workded at the Electrical Engineering Department of Khartoum University. In 1994 he was awarded a Ph.D. degree for work on the theory and modelling of single-electronic devices and systems. His current research interests concentrate on the simulation and modeling of FETs with emphasis on submicron gate length InGaAS channel pHEMTs for RF applications.

John Barker is Professor of Electronics in the Department of Electronics and Electrical Engi- neering. He has a long standing interest in computational methods, device modelling and transport theory. From $1970-85$ he was a member of the Theory group in the Dept. of Physics, University of Warwick, aside form 1978-79 when he worked at IBM T. J. Watson Laboratory, North Texas State University and Colorado State University. From 1987-89 he was academic director of the IBM UK/Glasgow University Kelvin Project on Numerically Intensive Parallel Computing. He is academic director of the Parallel Processing Centre at the University of Glasgow.

Steve Beaumont was educated at the University of Cambridge and has been with the Department of Electronics and Electrical Engineering at the University of Glasgow since 1978. He became Head of Department in 1995 and he convenes the Nanoelectronics Research Centre's management committee. His research interests lie in the field of nanmetre-scale fabrication and its application to electronic and optoelectronic devices. He has over 100 publications on electron beam nanolithography, dry etching, short-gate III-V based transistors, quantum transport devices, the optical properties of quantum dots, and single electron devices. Latterly he has become involved with the issue of manufacturability of $\mathrm{mm}$-wave circuits and the use of nanometre-scale fabrication techniques coupled with technology-based device simulations to forecast performance and yield with the minimum of process iterations. 

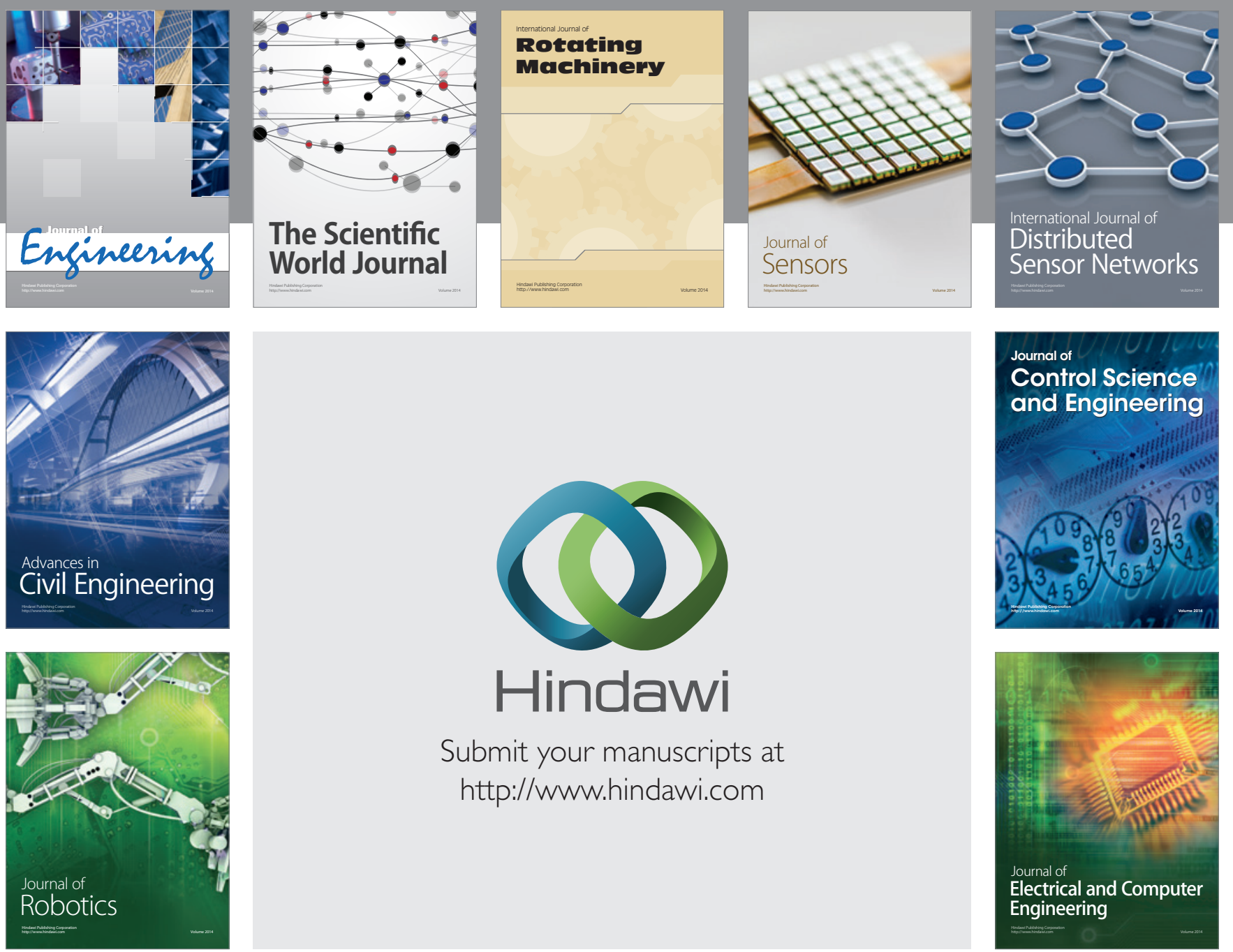

Submit your manuscripts at

http://www.hindawi.com
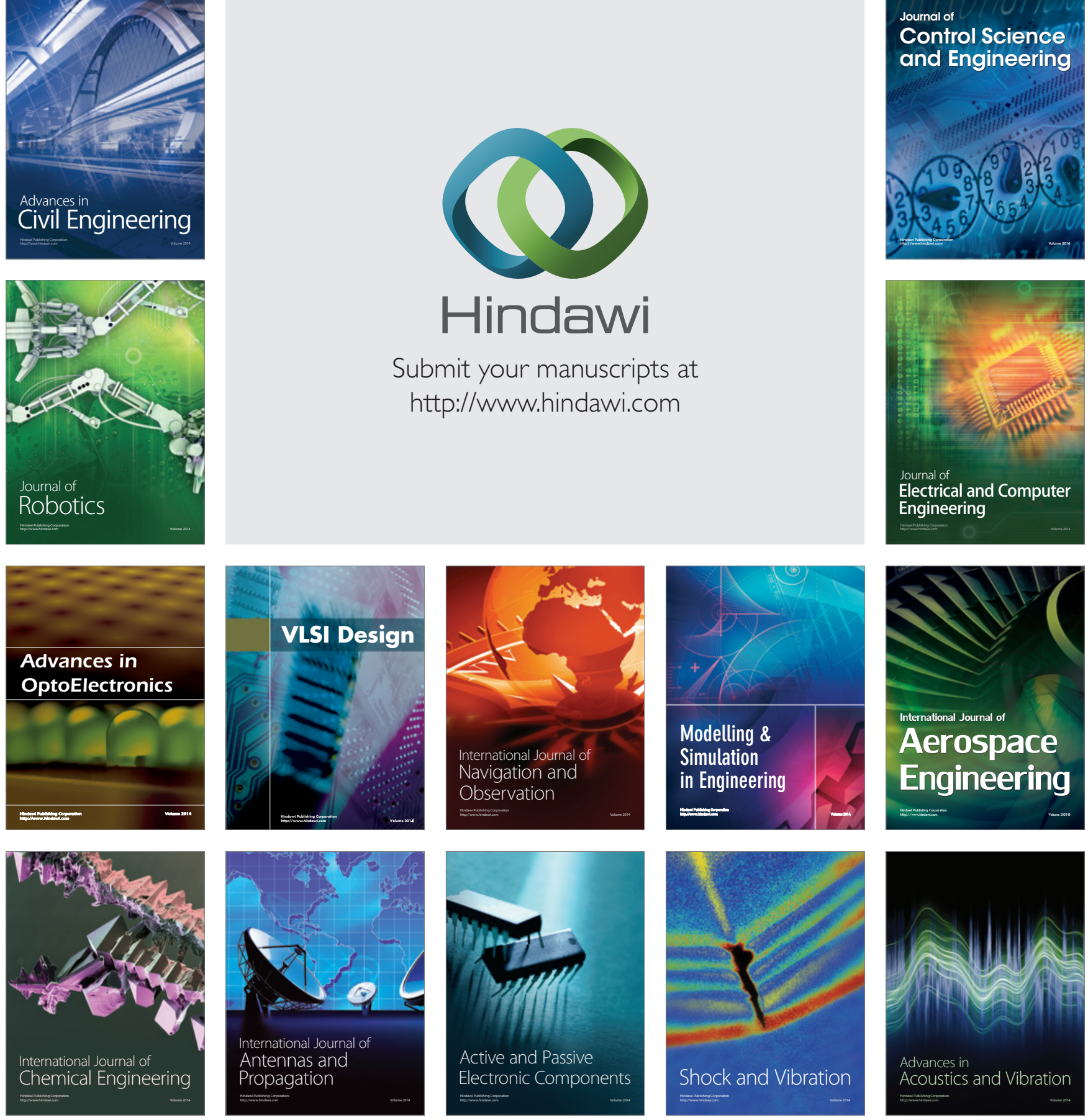